\title{
Anabases
}

ANABASES Traditions et réceptions de l'Antiquité

1 | 2005

Varia

\section{Giorgio CAMASSA, La lontananza dei Greci}

\section{Arnaldo Marcone}

\section{OpenEdition}

\section{Journals}

Edizione digitale

URL: http://journals.openedition.org/anabases/1506

DOI: 10.4000/anabases.1506

ISSN: 2256-9421

\section{Editore}

E.R.A.S.M.E.

\section{Edizione cartacea}

Data di pubblicazione: 1 marzo 2005

Paginazione: 299-301

ISSN: 1774-4296

Notizia bibliografica digitale

Arnaldo Marcone, « Giorgio camassa, La lontananza dei Greci », Anabases [Online], 1 | 2005, Messo online il 01 octobre 2011, consultato il 22 septembre 2020. URL : http://journals.openedition.org/anabases/ 1506 ; DOI : https://doi.org/10.4000/anabases.1506

Questo documento è stato generato automaticamente il 22 settembre 2020.

(c) Anabases 


\title{
Giorgio CAMASSA, La lontananza dei Greci
}

\author{
Arnaldo Marcone
}

\section{NOTIZIA}

Giorgio CAMASSA, La lontananza dei Greci, Roma, Edizioni Quasar, 2004, 109 p.

$13 €$ / ISBN 88-7140-261-8

1 Non siamo più Greci. Nessuno oggi penserebbe seriamente di ripetere con Shelley « we are all Greeks », o aderirebbe all'opinone di Wilhelm von Humboldt secondo il quale i Greci rappresentano l'ideale di quello che noi tutti dovremmo aspirare ad essere. G. Camassa in questo saggio affascinante di storia culturale ricostruisce il senso profondo del nostro attuale senso di alterità. Se i Greci si sono allontanati questo però non significa che siano assenti: forse "the Greeks are still very much with us ", come ha scritto di recente Robert Knox (The Oldest Dead White European Males, New York-London, 1993).

2 Il rapporto tra mondo moderno ed antico è stato un incessante confronto tra i Greci e i Romani esemplari e noi. Anzi si può dire che abbia interessato in primo luogo gli stessi Romani che si erano impadroniti della cultura greca prima di diventare a loro volta antichi e costituire quindi un modello (per l'« antico » nello stesso mondo antico si veda: S. Azzarà, “ Osservazioni sul senso delle rovine nella cultura antica ", in Senso delle rovine e senso dell'antico, a cura di W. Cuppari, Ann. Scuola Norm. Sup. di Pisa, Quad. 14, 2002, p. 1-12). Se nella prima età umanistica il parallelismo tra mondo antico e moderno riguardò sopratutto la sfera dell'etica, dal tardo Rinascimento in poi sono gli aspetti politici a prendere il sopravvento (in proposito la fortuna conosciuta dalla figura di Cicerone nei secoli è significativa: si veda ora E. Narducci, Cicerone e i suoi interpreti. Studi sull'Opera e la Fortuna, Pisa, 2004).

3 La ricerca delle analogie assunse una forma assai peculiare nella dotta Olanda del ‘600. A Leida, infatti, nel 1625 gli editori Elzevier iniziarono a pubblicare una serie di 
volumetti, di piccolo formato e scritti in latino, dal chiaro intento divulgativo, allo scopo di illustrare le repubbliche antiche e moderne, secondo un progetto, che era già stato di Grozio, di istituire una comparazione tra l'olanda, da un lato, Atene e Roma, dall'altro (cf. V. Conti, Consociatio civitatum. Le repubbliche nei testi elzeviriani, 1625-1649, Firenze, 1997). Lo stesso Meursius, d'altra parte, aveva insistito sul parallelismo tra l'Areopago ateniese e il senato veneziano: tanto in Olanda che a Venezia Atene aveva soppiantato Sparta nelle simpatie del pubblico colto. Intanto anche l'Inghilterra, all'approssimarsi della caduta della monarchia, riscopriva modelli repubblicani. Ci fu addirittura chi, come il giurista N. Bacon (A Historical Discourse of the Uniformity of the Government of England, 1647), arrivò a sostenere che non solo il governo degli antichi Britanni era stato repubblicano, ma che addirittura essi discendevano dai Greci, tanto simili era la loro forma di governo a quella di Atene e le loro istituzioni militari a quelle di Sparta.

4 La cultura del XVIII secolo è notoriamente percorsa da un eccezionale interesse per le antiche repubbliche greche e per Roma, considerate come una sorta di laboratorio per l'elaborazione di progetti di riforma politica e sociale. Proprio questa prospettiva spiega come l'attendibilità sul piano della ricostruzione storica e filologica cui si perveniva passasse di necessità in secondo piano.

5 Sparta, come G. Camassa puntualmente sottolinea, è un caso esemplare e le Rivoluzioni americana e francese sono un laboratorio straordinario. Il mito di Sparta è riutilizzato in America e in Francia dopo aver attraversato il mondo antico ed aver conosciuto un ritorno di interesse tra '500 e " 600 . Mably, come gli altri filolaconisti settecenteschi, si ispira, nella ricerca di un modello di costituzione ideale, alla presentazione che dell'organizzazione politica di Sparta faceva Plutarco nella Vita di Licurgo. Proprio questo mito era rovesciato poco tempo dopo dal pensatore che è giusto considerare, con Camassa, il capofila della reazione moderna all'idealizzazione dei Greci, Benjamin Constant. Non a caso Constant potè deridere Mably come colui il quale detestava la libertà individuale come si detesta un nemico personale.

6 Per Constant, « libertà » e indisturbato uso della ricchezza individuale sono sinonimi. E' questo il tema forte del ragionamento con cui egli respinge come oppressiva l'idea di libertà che gli antichi (specie Spartani) avevano praticato, e che i Giacobini, in tempi a lui vicini, avevano ripreso e rilanciato.

7 La storia del pensiero politico occidentale ha avuto nella democrazia ateniese un termine di riferimento costante talvolta esaltata come modello ideale, più spesso esecrata come un regime da evitare. In realtà, come è stato di recente sottolineato da Luciano Canfora (La democrazia. Storia di un'ideologia, Roma-Bari, 2004), e come Camassa suggerisce, la democrazia nasce sotto il segno di una contraddizione : in Grecia, come negli stessi Stati Uniti al momento della loro fondazione, l'ampiezza di diritti dei cittadini presuppone la schiavitù ; in Francia, a partire dalla rivoluzione del 1789 e, quindi, nel mondo occidentale la democrazia è costantemente in bilico tra giacobinismo e bonapartismo. Forse non è un caso se fu proprio il Bonaparte a farsi promotore di edizioni di "Stato" dei classici greci, come la Geografia di Strabone. Viceversa Condorcet, nell'aprile del 1792, in un momento in cui pure agli autori antichi si guardava come paradigmi di valore assoluto, in un Rapport all'Assemblea Legislativa aveva sentenziato che per i grandi scrittori del passato erano ormai disponibili delle traduzioni. 
Pochi poeti hanno sentito il fascino del mondo ellenico come Friedrich Hölderlin. Ebbene la lettura delle sue liriche (se ne veda l'eccellente edizione con commento di Luigi Reitani, Milano 2001) ci può dare una sorpresa. In « Grecia », una poesia giovanile, tutto sommato di maniera, il mondo ellenico è celebrato secondo i canoni dell'idealizzazione classicistica. Ben più complessa e originale è l'ispirazione di " Arcipelago ». Qui si canta la gloria di Atene, intesa come una comunità organicistica fondata su valori autenticamente religiosi, con il rimpianto di un passato irrimediabilmente perduto, contrapposto ad un presente dove predomina l'individualismo : «Dimmi dov'è Atene? E' forse crollata nella cenere, sulle urne dei maestri, Dio in lutto, la tua città, la più amata da te, sulle tue rive ? " (vv. 62-64, trad. di L. Reitani).

9 A fronte di tanti «antichi ", spesso falsi perché sentiti o voluti troppo vicini e quindi inevitabilmente falsificati, una matura e consapevole consapevolezza della lontananza dei Greci può rappresentare un favorevole punto di partenza per studiarli e capirli nella loro preziosa originalità (per una proposta di cercare nell'antico non l'identico ma il diverso si veda S. Settis, Futuro del «classico», Torino 2004 con le osservazioni di V. Saladino, in Il Ponte 60, 2004, p. 106-115). Categorie come quelle di tempo e di spazio, cui nel libro di Camassa sono dedicati due capitoli significativi, sono particolarmente importanti (in proposito si vedano anche le considerazioni di M. Bretone, Diritto e tempo nella tradizione europea, Roma-Bari, 2004).

Merita, per sintetizzare il tema di fondo di questo libro, ridare la parola a Hölderlin che, nella famosa lettera inviata all'amico Böhlendorff all'inizio di dicembre del 1801, poco prima della partenza per Bordeaux, scrive (cito da M. Heidegger, La poesia di Hölderlin, trad. it., Milano, 1988, p. 107) : «Non c'è cosa che apprendiamo con più difficoltà di questo: servirci liberamente di ciò che appartiene alla nostra nazione. E, come credo, è proprio la chiarezza della rappresentazione che è per noi così naturale come lo è per $\mathrm{i}$ Greci il fuoco del cielo. Ma il proprio deve venire appreso esattamente come l'estraneo. Per questo i Greci sono per noi indispensabili. Solo che precisamente in ciò che abbiamo di proprio, di nazionale, noi non terremo loro dietro, perché, come dicevo, il servirsi liberamente di ciò che è proprio è la cosa più difficile di tutte ».

11 Dobbiamo essere grati a G.Camassa per averlo dimostrato in maniera tanto accattivante e persuasiva.

\section{AUTORI}

\section{ARNALDO MARCONE}

Università di Udine 